\title{
Predicting health-related quality of life (EQ- 5D-5 L) and capability wellbeing (ICECAP-A) in the context of opiate dependence using routine clinical outcome measures: CORE- OM, LDQ and TOP
}

\author{
Jasmine Peak ${ }^{1}$, Ilias Goranitis ${ }^{6}$, Ed Day ${ }^{2,3}$, Alex Copello $^{2,4}$, Nick Freemantle ${ }^{5}$ and Emma Frew ${ }^{1 *}$ (D)
}

\begin{abstract}
Background: Economic evaluation normally requires information to be collected on outcome improvement using utility values. This is often not collected during the treatment of substance use disorders making cost-effectiveness evaluations of therapy difficult. One potential solution is the use of mapping to generate utility values from clinical measures. This study develops and evaluates mapping algorithms that could be used to predict the EuroQol-5D (EQ-5D-5 L) and the ICEpop CAPability measure for Adults (ICECAP-A) from the three commonly used clinical measures; the CORE-OM, the LDQ and the TOP measures.

Methods: Models were estimated using pilot trial data of heroin users in opiate substitution treatment. In the trial the EQ-5D-5 L, ICECAP-A, CORE-OM, LDQ and TOP were administered at baseline, three and twelve month time intervals. Mapping was conducted using estimation and validation datasets. The normal estimation dataset, which comprised of baseline sample data, used ordinary least squares (OLS) and tobit regression methods. Data from the baseline and three month time periods were combined to create a pooled estimation dataset. Cluster and mixed regression methods were used to map from this dataset. Predictive accuracy of the models was assessed using the root mean square error (RMSE) and the mean absolute error (MAE). Algorithms were validated using sample data from the follow-up time periods.
\end{abstract}

Results: Mapping algorithms can be used to predict the ICECAP-A and the EQ-5D-5 L in the context of opiate dependence. Although both measures can be predicted, the ICECAP-A was better predicted by the clinical measures. There were no advantages of pooling the data. There were 6 chosen mapping algorithms, which had MAE scores ranging from 0.100 to 0.138 and RMSE scores ranging from 0.134 to 0.178 .

Conclusion: It is possible to predict the scores of the ICECAP-A and the EQ-5D-5 $L$ with the use of mapping. In the context of opiate dependence, these algorithms provide the possibility of generating utility values from clinical measures and thus enabling economic evaluation of alternative therapy options.

Trial registration: ISRCTN22608399. Date of registration: 27/04/2012. Date of first randomisation: 14/08/2012.

Keywords: ICECAP, EQ-5D, Mapping, Addiction, Mental health, Preference-based measures, Condition-specific measures, Economic evaluation

\footnotetext{
* Correspondence: E.Frew@bham.ac.uk

${ }^{1}$ Health Economics Unit, Institute of Applied Health Research, Public Health

Building, University of Birmingham, B15 2TT, Birmingham, UK

Full list of author information is available at the end of the article
}

(c) The Author(s). 2018 Open Access This article is distributed under the terms of the Creative Commons Attribution 4.0 International License (http://creativecommons.org/licenses/by/4.0/), which permits unrestricted use, distribution, and reproduction in any medium, provided you give appropriate credit to the original author(s) and the source, provide a link to the Creative Commons license, and indicate if changes were made. The Creative Commons Public Domain Dedication waiver (http://creativecommons.org/publicdomain/zero/1.0/) applies to the data made available in this article, unless otherwise stated. 


\section{Background}

In many healthcare systems around the world, resources are scarce and the demand for healthcare outweighs supply. This scarcity warrants the need for economic evaluation to aid decision makers with information about the most efficient use of resources in order to maximise the health gained for every unit of currency spent. Within the UK, and in many other country jurisdictions, the most common approach to economic evaluation is the cost-utility analysis. The outcomes of a cost-utility analysis are expressed in quality-adjusted life years (QALYs) [1]. QALYs take into account both quality and length of life and offer a commensurate unit that allows comparisons of cost-effectiveness across different disease areas and interventions [2]. To measure QALYs, generic preference-based measures of health-related quality of life (HRQOL) are required [3] to capture a broad construct of health through key dimensions that are known to affect quality of life [4]. Commonly, the EuroQol-5D (EQ-5D-5 L) measure is used in economic evaluations to estimate QALYs [5]. The EQ-5D-5 L describes HRQOL through the dimensions of mobility, self-care, usual activities, pain and discomfort, and anxiety and depression.

When considering substance use disorders, the broader concept of wellbeing is considered more appropriate to reflect the clinical and policy objectives [6]. Drug dependence undermines an individual's capability [6], and this disempowerment is largely overlooked in the health economics of addiction-related interventions as a result of the narrow definition of HRQOL. Until recently, it was hard to define and quantify wellbeing for the purposes of an economic evaluation. Amartya Sen's work on capabilities allowed for a conceptualization of wellbeing through human functionings (what an individual 'does') and capabilities (the ability of the individual to exercise a functioning) [7]. The development of the ICEpop CAPability measure for Adults (ICECAP-A) based on Amartya Sen's capability approach, means that wellbeing can now be measured in a way that is compatible for use in economic evaluations [8]. The ICECAP-A measures capability wellbeing through the dimensions of stability, enjoyment, achievement, attachment and autonomy. Both EQ-5D-5 L and ICECAP-A were found to have the appropriate construct validity within the addiction context, but ICECAP-A appeared to be significantly more responsive to changes of key clinical indicators [9].

In order to reduce the burden of assessment on patients and to help acquire data for clinical means, particularly in the context of substance use disorder, studies tend to rely only on clinical context-specific measures. For substance use disorders, measures that are commonly used to assess the level and impact of dependence and assess the effectiveness of a treatment are the Clinical Outcomes in Routine Evaluation - Outcome Measure (CORE-OM), the
Leeds Dependence Questionnaire (LDQ), and the Treatment Outcomes Profile (TOP). The CORE-OM and the TOP are instruments that are used to assess the treatment outcome, whilst the LDQ is used to assess the level of dependence at the time of assessment $[10,11]$. These measures, however, are unsuitable for use within health economic evaluations [12]. To enable information from these measures to be used in economic evaluations, a process called 'mapping' can be applied [13]. Mapping, quantifies the relationship between different measures using appropriate statistical techniques [14, 15], and allows for the estimation of HRQOL and wellbeing for use in economic evaluations using data collected from routine clinical measures.

This study aims to map three clinical instruments that are often used in the routine care of addiction and opiate dependence (CORE-OM, LDQ, and TOP) onto the EQ-5D-5 L and ICECAP-A measures, generating algorithms that can be used in future studies to aid reimbursement decisions in the absence of information related to EQ-5D-5 L and ICECAP-A. With these mapping algorithms, data from the three clinical measures can be translated into health and capability scores for use in an economic evaluation. The mapping algorithms were developed using data from a pilot randomised control trial (RCT) that sought to explore the effectiveness of two psychosocial interventions for heroin users receiving opiate substitution treatment (OST) in England [10]. This is the first study to develop mapping algorithms from routine clinical outcome measures used in addiction therapy onto the EQ-5D-5 L and ICECAP-A.

\section{Methods}

The study uses data collected as part of a pilot randomized controlled trial designed to investigate the clinical and cost-effectiveness of two psychological interventions delivered in addition to the usual care of individuals who had been receiving opiate substitution treatment for more than one year [10]. All trial participants met the ICD-10 criteria for opioid dependence and were recruited if they were in opiate substitution treatment with methadone or buprenorphine for more than a year but still reported heroin use during the last month. The only exclusion criteria were having a physical or mental health condition that prevented engagement in the psychosocial intervention, or an imminent period of imprisonment. A number of client outcomes, including mental health (CORE-OM), substance dependence (LDQ), physical and psychological health (TOP), health-related quality of life (EQ-5D-5 L), and capability wellbeing (ICECAP-A) were assessed at baseline, 3 months and 12 months post-randomisation. These outcome measures are described in detail below. The trial was conducted by three community drug teams in England. All 
trial participants provided written informed consent, and ethical approval was obtained from the Black Country NHS Research Ethics Committee (reference: 12/WM/0046).

\section{Outcome measures \\ Clinical outcomes in routine evaluation - outcome measure (CORE-OM)}

The CORE-OM is widely used to assess the mental health effects of psychological interventions [16]. It comprises 34 items across four main areas of focus: subjective wellbeing, symptoms, functioning and risk. A five point rating scale has been adopted, with 0 representing not at all, whilst 4 represents all the time [17]. A mean item score is commonly generated to allow an understanding of the level of psychological distress of an individual. The CORE-OM is a widely used measure that generates clinically meaningful information [17], and it has been regarded as acceptable, reliable, valid [16].

\section{Leeds dependence questionnaire (LDQ)}

The LDQ is used to identify an individual's level of dependence on a variety of substances [18]. It features 10 items that are rated on a scale from 0 to 3; 0 representing never, whilst 3 represents nearly always. The questions are centered around substance use and frequency; asking about desires, how substances fit into daily routines and any future plans of taking substances [19]. The scores of all items are aggregated to indicate the overall level of dependence. This can vary between 0 and 30 with the cut-offs of 10 and 22 used to classify individual's level of dependence into low, moderate, and severe. Raistrick et al. [18] describe a variety of features of LDQ that suggest it may be able to complement economic evaluation. The authors conclude that LDQ is understandable and sensitive to change in the level of dependency over time and across all substance dependencies.

\section{Treatment outcomes profile (TOP)}

The TOP is used to assess the change and progress in key areas of life for individuals who are being treated for drug or alcohol addiction [20]. It features 20 questions, reflecting the four domains of substance use- injecting risk behaviour, crime and health and social care functioning [21]. For this study, the health and social care functioning aspect, which included psychological health, physical health and the overall quality of life dimensions were of importance. These three dimensions are rated on a scale of 0 (which represents poor) to 20 (which represents good) [20].

\section{EuroQol - 5 dimensions - 5 levels (EQ-5D-5 L)}

The EQ-5D-5 L is an instrument that is used to measure health-related quality of life. It is a self-reported questionnaire that covers five dimensions of health; mobility, self-care, usual activities, pain and discomfort, and anxiety and depression. Participants select their functioning level from five options ranging from 1 to 5 , with 1 representing that an individual has no functioning problems in a given dimension, whereas 5 represents severe problems with functioning [22]. An index of health-related quality of life is generated to illustrate an individual's overall health status using a population tariff. This study used the English population tariff which was developed based on the time trade-off and discrete choice experiment methods [23]. The health index score ranges from -0.281 to 1 , with negative values representing health states worse than death, 0 representing the "dead" state, and 1 the "full health" state. The reliability and validity of the EQ-5D-5 L for use with the population of study in this investigation has already been published [9].

\section{ICEpop CAPability measure for adults (ICECAP-A)}

The ICECAP-A is a measure of capability wellbeing. It focuses on ability to function across five key dimensions of wellbeing. These are stability, enjoyment, achievement, attachment and autonomy. Participants select their capability level from four options ranging from 1 to 4 , with 1 representing that an individual has limited capability in a given dimension, whereas 4 represents high levels of capability [8]. An index of capability wellbeing is generated that illustrates an individual's overall capability levels from 0 to 1 using a UK population tariff developed based on the best-worst scaling method [24]. A score of 0 suggests that an individual has no capability, whilst 1 represents full capability [8]. The reliability and validity of the ICECAP-A for use with the population of study in this investigation has also been published [9].

\section{Estimation and validation datasets}

A common approach across mapping studies is to split the dataset into an estimation dataset, where the mapping algorithm between the source outcome measures (COREOM, LDQ and TOP) and the target measures (EQ-5D-5 L and ICECAP-A) is first derived, and a validation dataset, where the predictive properties of the algorithm are tested [25]. Two approaches were used to determine the estimation and validation dataset. In the first approach, the estimation sample was developed from the baseline data and the validation sample from the 3 month follow-up data. In the second approach, the data from the baseline assessment and 3 month follow up were pooled in order to create a larger estimation sample, and the 12 month follow up data were then used for validation purposes, similar to other studies in the literature [26-28].

\section{Statistical analysis}

Longworth and Rowen [25] highlight a variety of regression methods that are often used in mapping studies. 
The type of method employed depends on whether the prediction goal is for the overall index score or the dimension scores of a preference-based measure. Given that mapping onto an overall index score rather than dimension scores has been found to offer better predictive ability [29], this approach was adopted for the purposes of this study.

In the first mapping approach, ordinary least squares (OLS) and tobit regressions were used. The OLS regression approach is commonly used in mapping studies [25] and has been regarded as robust at predicting the mean index score of a preference-based measure [30]. The tobit regression is a censored regression method providing opportunity to limit predictions within the appropriate range of scores for EQ-5D-5 L (- 0.281 to 1) and ICECAP-A (0 to 1$)$. Given that the OLS regression is likely to provide predictions beyond these ranges, these predictions were subsequently forced to the appropriate threshold value. This is.

a common approach in mapping studies [31-34]. In the second mapping approach, a cluster regression and a multilevel mixed effects regression at an individual level were used to account for within-subject dependence [35, 36].

A number of potential explanatory variables were available and these were explored incrementally and in line with the recommended methods guidance provided by Longworth and Rowen [25]. These included overall scores, dimension scores, quadratic terms for potentially nonlinear relationships, interaction terms, and patient characteristics (i.e. age and gender). All model specifications used to map from CORE-OM, LDQ and TOP onto the EQ-.

5D-5 $\mathrm{L}$ and ICECAP-A are shown in Table 1.

To test whether the algorithms were fit for purpose, the $R^{2}$, the adjusted $R^{2}$, the Akaike information criterion (AIC) and the Bayesian information criterion (BIC) were assessed. For the final model choice, Brazier et al. [14] argue that predictive ability and not fit should be considered, and therefore an internal and external validation of the models' predictive ability was undertaken. Internal validation involved the prediction of the EQ-5D-5 L and ICECAP-A index scores from each model's outputs and evaluated how close the predicted results were to the observed ones in the estimation dataset using the root-mean-squared-error (RMSE) and the mean absolute error (MAE) [25]. For external validation, models' coefficients were applied to the scores of CORE-OM, LDQ, and TOP in the validation dataset and the results were plotted on a graph in order to see how close the predicted EQ-5D-5 L and ICECAP-A index scores were to the actual index scores using the RMSE and MAE. All analyses were undertaken in Stata version 13MP.

\section{Results}

\section{Descriptive statistics}

Table 2 presents the demographic details for the 83 trial participants. The mean age was 37 years and the sample comprised mostly men (87\%). The majority were of white ethnicity (84\%) and unemployed (79\%). Nearly $82 \%$ of the sample received some form of state benefits. At baseline, mean capability (ICECAP-A) index score was $0.662(\mathrm{SD}=0.189)$ and mean health $(\mathrm{EQ}-5 \mathrm{D}-5 \mathrm{~L})$ index score was $0.806(\mathrm{SD}=0.204)$. The summary statistics for the two preference-based outcome measures and for both the estimation and validation samples across the different follow-up periods are shown in Additional file 1: Table S1.

\section{Mapping CORE-OM onto EQ-5D-5 L and ICECAP-A}

The performance of the different models in the internal (estimation) and external validation samples is provided in Additional file 2: Table S2 and Additional file 3: Table S3, respectively. The results showed that most models

Table 1 Summary of the model specifications used when mapping from CORE-OM, LDQ, and TOP onto EQ-5D-5 L and ICECAP-A

\begin{tabular}{|c|c|c|c|}
\hline Model & CORE-OM & LDQ & TOP \\
\hline 1 & Mean score & Aggregate score & Overall quality of life score \\
\hline 2 & $\begin{array}{l}\text { Mean score; } \\
\text { Mean score }\end{array}$ & $\begin{array}{l}\text { Aggregate score; } \\
\text { Aggregate score }^{2}\end{array}$ & $\begin{array}{l}\text { Overall quality of life score; } \\
\text { Physical and Psychological } \\
\text { health status }\end{array}$ \\
\hline 3 & $\begin{array}{l}\text { Wellbeing; Symptoms; } \\
\text { Functioning; Risk }\end{array}$ & $\begin{array}{l}\text { Best model from } \\
\text { above plus Age and } \mathrm{Age}^{2}\end{array}$ & Model 2 plus quadratic terms \\
\hline 4 & $\begin{array}{l}\text { Model } 3 \text { plus } \\
\text { quadratic terms }\end{array}$ & Model 3 plus Gender & Model 3 plus interaction terms \\
\hline 5 & $\begin{array}{l}\text { Model } 4 \text { plus } \\
\text { interaction terms }\end{array}$ & & $\begin{array}{l}\text { Best model from above } \\
\text { plus Age and } \mathrm{Age}^{2}\end{array}$ \\
\hline 6 & $\begin{array}{l}\text { Best model from } \\
\text { above plus Age and } \mathrm{Age}^{2}\end{array}$ & & Model 5 plus Gender \\
\hline 7 & Model 6 plus Gender & & \\
\hline
\end{tabular}

CORE-OM Clinical Outcomes in Routine Evaluation - Outcome Measure, LDQ Leeds Dependence Questionnaire, TOP Treatment Outcomes Profile, EQ-5D-5 L EuroQol - 5 Dimensions - 5 Levels, ICECAP-A ICEpop CAPability measure for Adults 
Table 2 Patient Demographic Information

\begin{tabular}{ll}
\hline Number in Study & 83 \\
\hline Age, mean (SD) & $37.1(6.40)$ \\
Men, $\mathrm{n}(\%)$ & $72(86.80)$ \\
White, $\mathrm{n}(\%)$ & $70(84.30)$ \\
Employed, $\mathrm{n}(\%)$ & $17(20.50)$ \\
Married, $\mathrm{n}(\%)$ & $2(2.40)$ \\
Family Accommodation, $\mathrm{n}(\%)$ & $75(90.40)$ \\
Secondary Education or less, $\mathrm{n}(\%)$ & $56(67.50)$ \\
State Benefit Recipients, $\mathrm{n}(\%)$ & $68(81.90)$ \\
\hline
\end{tabular}

$n$ number of patients, $S D$ standard deviation

predicted the EQ-5D-5 L and ICECAP-A index scores well in both samples and for both estimation approaches. For the EQ-5D-5 L most models provided predictions within a 0.03 range from the observed health index score, and therefore not different in terms of clinical importance [37]. Exceptions were mainly from the tobit regressions. For the ICECAP-A, most models provided predictions within a 0.01 range from the observed capability index scores. In the internal validation sample, model specification 5 , which included the CORE-OM dimension scores plus quadratic and interaction terms, consistently produced the lowest RMSE and MAE scores across the different types of regression and the largest variability around the predicted mean EQ-5D-5 L and ICECAP-A index scores. Because of this variability, Model 5 resulted in large RMSE and MAE in the external validation sample. In this sample, model specification 3, which included the four CORE-OM dimensions as covariates, produced consistently the lowest RMSE and MAE results for the EQ-5D-5 L at the mean value as well as at repeated measurements (i.e. 25th, 50th, and 75th percentiles), whilst model specification 2, which included the mean CORE-OM score and its squared term, showed the best performance across the different models and estimation approaches (Additional file 4 Table S4). In terms of health index score, the OLS model 3 had the lowest RMSE (0.134) and MAE (0.1) and predicted the mean EQ-5D-5 L index score with $\mathrm{a}<0.007$ deviation from the observed score (0.83). In terms of the capability index score, tobit model 2 was found to have the best predictive properties with RMSE and MAE scores of 0.138 and 0.106 respectively. The coefficients for each model covariate and the model's fit for the two mapping algorithms are shown in Table 3. Figures 1 and 2 show graphs displaying the predicted scores in comparison to the observed scores for the chosen algorithms, when mapping from the CORE-OM. The ICECAP-A is better predicted.
Table 3 Mapping Models from the CORE-OM to the EQ-5D-5 L and the ICECAP-A

\begin{tabular}{lll}
\hline & EQ-5D-5 L & ICECAP-A \\
\hline Model & OLS (3) & Tobit (2) \\
Intercept & $1.048^{\mathrm{c}}$ & $0.999^{\mathrm{c}}$ \\
CORE-OM score & & $-0.296^{\mathrm{a}}$ \\
CORE-OM score ${ }^{2}$ & & 0.041 \\
Wellbeing & 0.0005 & \\
Symptoms & $-0.109^{\mathrm{c}}$ & \\
Functioning & -0.010 & \\
Risk & -0.033 & -69.824 \\
AIC & -56.362 & -60.296 \\
BIC & -44.451 & -1.197 \\
Adjusted R ${ }^{2}$ Pseudo R & 0.355 & 0.138 \\
RMSE (external sample) & 0.134 & 0.106 \\
MAE (external sample) & 0.100 & \\
\hline aStatistically significant at the $1 \%$ level & AIC Akaike information criterion
\end{tabular}

${ }^{a}$ Statistically significant at the $1 \%$ level. AIC Akaike information criterion, $B I C$ Bayesian information criterion, MAE mean absolute error, OLS ordinary least squares, RMSE root mean squared error

Using CORE-OM as an example, the EQ-5D-5 L utility score can be calculated from the following coefficients:

$$
\begin{aligned}
E Q-5 D-5 L \text { utility score }= & 1.048+(\text { Wellbeing }) \\
& \times(0.0005)-(\text { Symptoms }) \\
& \times(0.109)-(\text { Functioning }) \\
& \times(0.010)-(\text { Risk })(0.033)
\end{aligned}
$$

\section{Mapping LDQ onto EQ-5D-5 L and ICECAP-A}

Detailed information about the performance of the different models used to predict health and capability index scores in the internal and external validation samples is provided in the Additional file 5: Table S5 and Additional file 6: Table S6. Similar to the CORE-OM measure, the results indicated that most models predicted the EQ-5D-5 L and ICECAP-A index scores closely in both samples and for both estimation approaches. The only exception was from the tobit models, which gave predictions beyond the potentially acceptable 0 . 03 threshold difference from the observed EQ-5D-5 L index scores. For both internal and external samples, model specification 4, which included the total LDQ score, age, age squared and sex, was found to offer the best predictive ability, with RMSE and MAE ranging between 0.172-0.216 and 0.122-0.146 across the different analyses for the health index score and between $0.163-0.194$ and $0.133-0.154$ for the capability index score. OLS model 4 when mapping to the EQ-5D-5 L had the better predictive ability. The model produced a MAE score of 0.128 and RMSE score of 0.178 . OLS model 4 also had better predictive ability when mapping to the ICECAP-A. This model produced a MAE score of 0.138 and a RMSE score of 0.171 


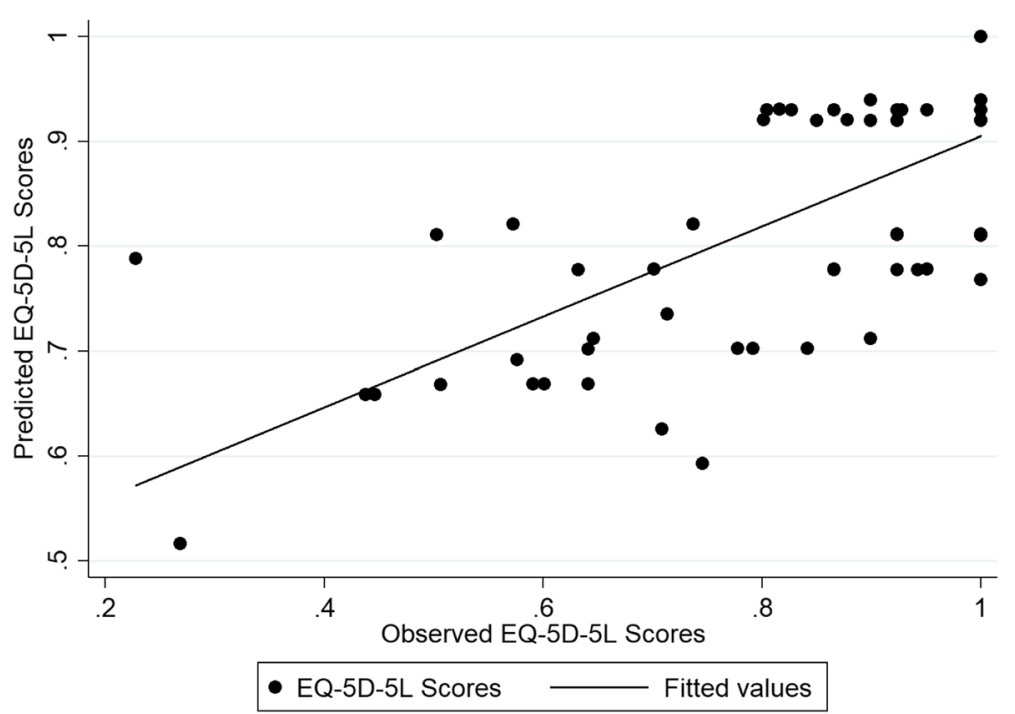

Fig. 1 The observed vs predicted scores of the EQ-5D-5 L mapped from the CORE-OM based on Model 3

(Additional file 7 Table S7). The coefficients for each model covariate based on the external validation sample and the model's fit are detailed in Table 4. Figures 3 and 4 show the difference between the EQ-5D-5 L and the ICECAP-A predictions.

The EQ-5D-5 L scores were more dispersed and spread than the ICECAP-A scores.

\section{Mapping from the TOP onto EQ-5D-5 L and ICECAP-A}

The performance of the different mapping algorithms from the TOP measure onto EQ-5D-5 L and ICECAP-A measures in both internal and external validation samples is shown in the Additional file 8: Table S8 and Additional file 9 Table S9. Most models predicted the EQ-5D-5 L and
ICECAP-A index scores well in both samples and for both approaches. Tobit models gave again predictions of health index scores that were more than 0.03 points different to the observed EQ-5D-5 L scores but performed well in terms of predicting capability scores. Model specifications 4; which included TOP dimension scores, quadratic terms and interaction terms and model 6 , which included the covariates in model 4 with the addition of age, age ${ }^{2}$ and gender; appeared to have a better performance in the internal sample resulting in RMSE and MAE that ranged between $0.155-0.199$ and $0.122-0.126$ respectively but again with larger variability around the predicted mean index scores. The model specification with the best external predictive ability across the different models and

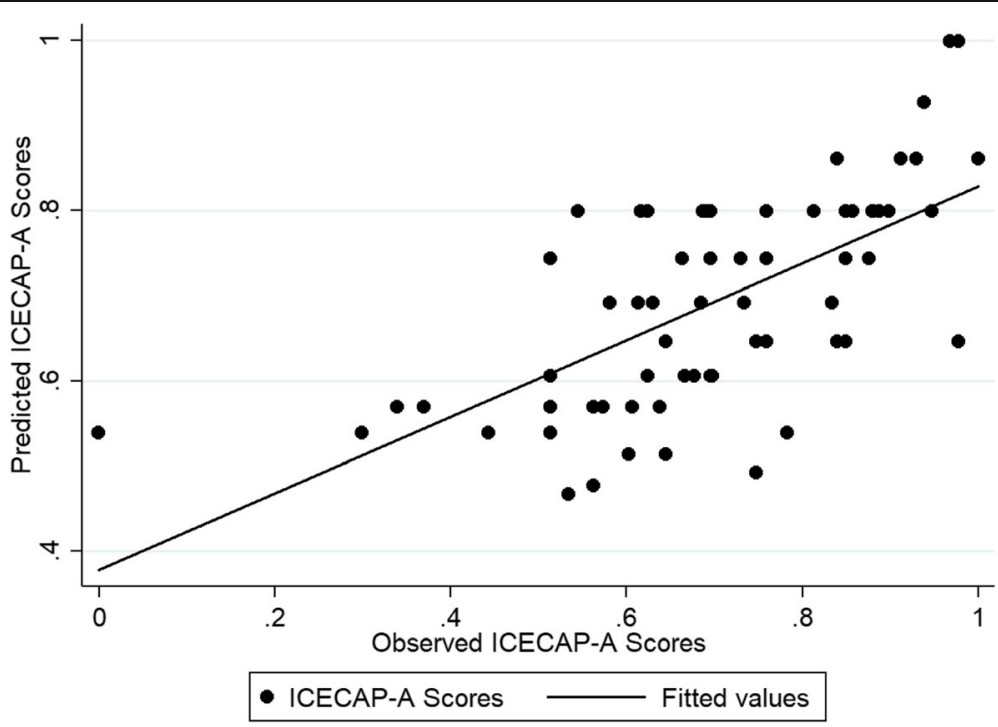

Fig. 2 The observed vs predicted scores of the ICECAP-A mapped from the CORE-OM based on Model 2 
Table 4 Mapping Models from the LDQ to the EQ-5D-5 L and the ICECAP-A

\begin{tabular}{lll}
\hline & EQ-5D-5 L & ICECAP-A \\
\hline Model & OLS (4) & OLS (4) \\
Intercept & 0.415 & $0.958^{\mathrm{a}}$ \\
LDQ score & $-0.014^{\mathrm{b}}$ & $-0.0122^{\mathrm{b}}$ \\
Age & 0.033 & -0.004 \\
Age $^{2}$ & -0.0005 & -0.000002 \\
Sex (if Female) & -0.016 & -0.052 \\
AIC & -44.943 & -53.753 \\
BIC & -32.971 & -41.781 \\
Adjusted R & 0.250 & 0.214 \\
RMSE (external sample) & 0.178 & 0.171 \\
MAE (external sample) & 0.128 & 0.138
\end{tabular}

${ }^{\mathrm{a} S t a t i s t i c a l l y ~ s i g n i f i c a n t ~ a t ~ t h e ~} 5 \%$ level; ${ }^{\mathrm{b}}$ Statistically significant at the $1 \%$ level. AIC Akaike information criterion, BIC Bayesian information criterion, MAE mean absolute error, OLS ordinary least squares, RMSE root mean squared error

for both EQ-5D-5 L and ICECAP-A was model specification 2, which included the three TOP dimensions only (Overall quality of life score, Physical health status, and Psychological health status). Overall, the OLS model 2 predicted the observed EQ-5D-5 L (0.83) and ICECAP-A (0.69) index score with a -0.01 point difference. For EQ5D-5 L and ICECAP-A, the RMSE scores were 0.167 and 0.151 respectively, while the MAE scores were 0.123 for both mapping algorithms (Additional file 10: Table S10). The coefficients for each model covariate based on the external validation sample and the models' fit are detailed in Table 5. Figures 5 and 6 display the graphs detailing the predicted and observed scores when mapping from the TOP. The EQ-5D-5 L results had a greater dispersion, and were plotted further away from the fitted value line.

\section{Discussion}

Policy decision makers are becoming more focused on treatment outcomes that go beyond consideration of abstinence alone and capture wider treatment impact upon patients HRQOL [38]. These measures should include economic measures that allow consideration of value for money. This study developed mapping algorithms from three key clinical measures in the context of opiate dependence (CORE-OM, LDQ, and TOP) onto the EQ5D-5 L and ICECAP-A, which are recommended by the National Institute for Health and Care Excellence (NICE) in the economic evaluation of health and social care interventions [5]. These algorithms introduce the possibility of estimating HRQOL and capability wellbeing from the information contained within each clinical measure and therefore the ability to make treatment recommendations based on wider quality of life and wellbeing outcomes. As these instruments are focused exclusively upon health-related quality of life, and wellbeing effects, these algorithms provide the vehicle to target resources towards treatment that will benefit population quality of life. The ICECAP-A was better predicted than the EQ-5D-5 L. This suggests that when mapping from the clinical measures to the ICECAP-A, there will be a greater alignment between the wellbeing aspects than when mapping to the EQ-5D-5 L. This presents an interesting development for reimbursement decisions within the context of opiate dependence and OST.

This is the first study to generate mapping algorithms for these clinical measures. This was a particularly difficult hard to reach population under study with many participants receiving OST at a therapeutic dose for at least 5 years, and still reporting heroin use [11]. The study benefited from good completion rates across the

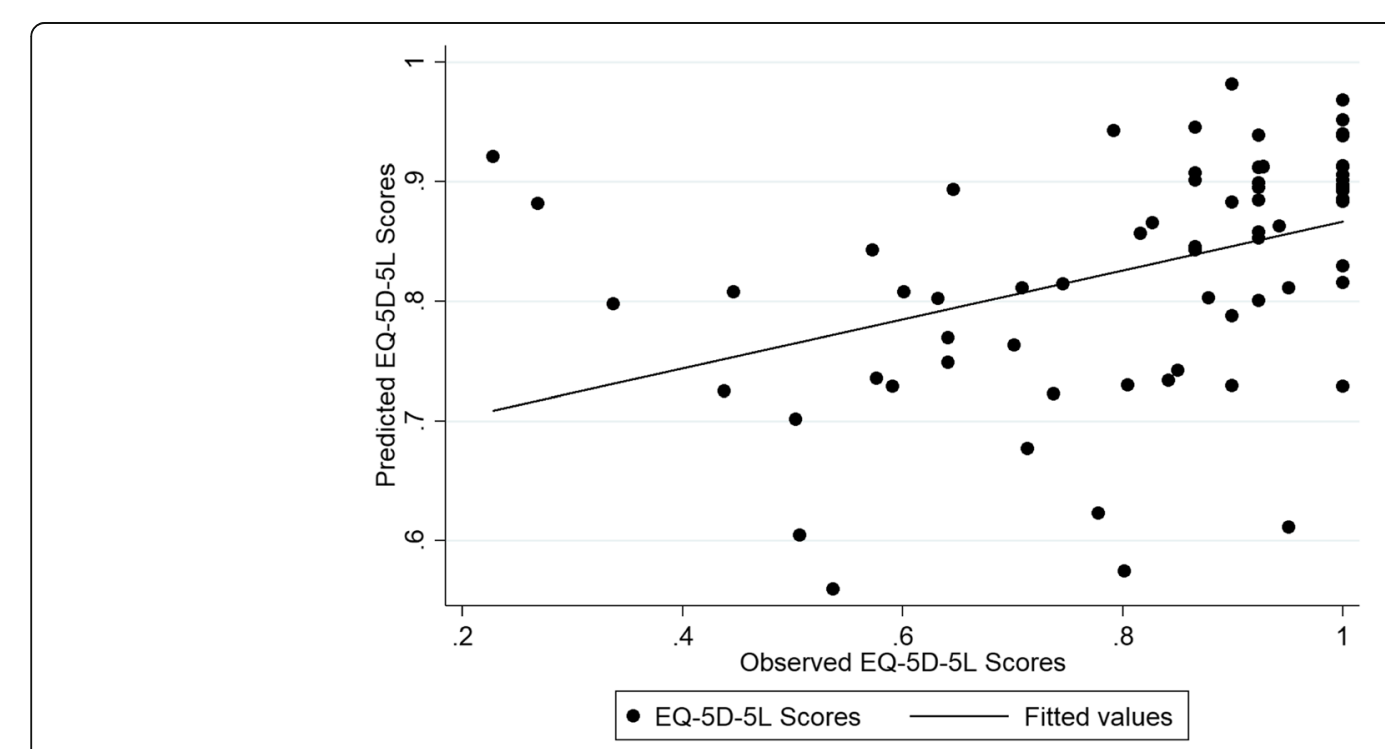

Fig. 3 The observed vs predicted scores of the EQ-5D-5 L mapped from the LDQ based on Model 4 


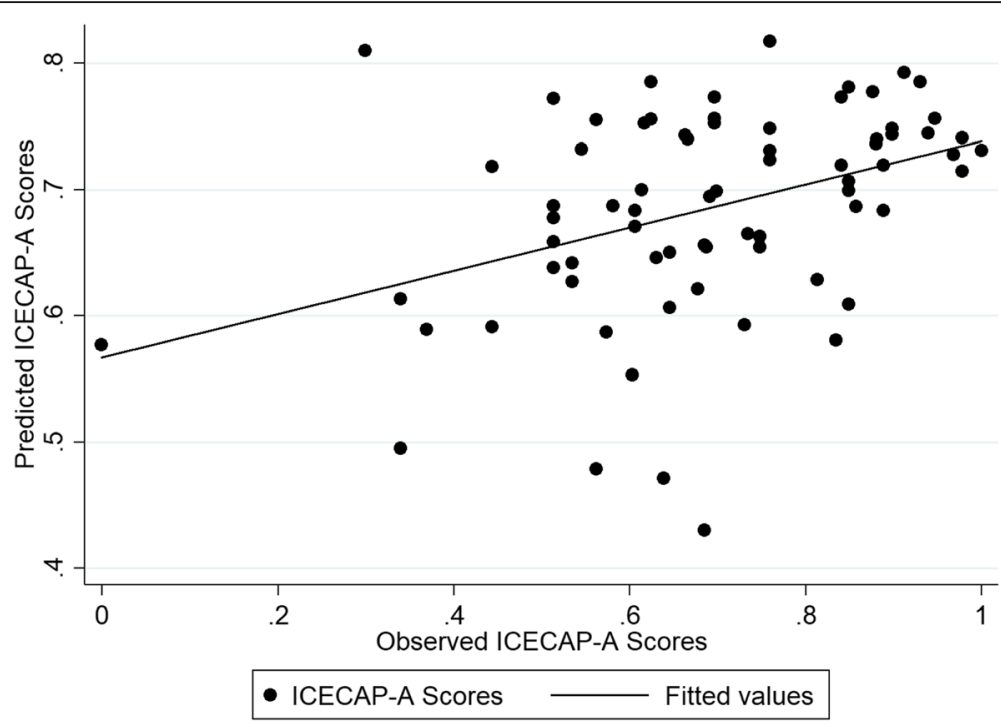

Fig. 4 The observed vs predicted scores of the ICECAP-A mapped from the LDQ based on Model 4

different measures, and although the primary trial focused on a hard to reach population undergoing opiate substitution treatment but still reporting heroin use, the distribution of health and capability scores provides some confidence that these algorithms are likely to be generalizable to other contexts involving substance use disorders. The algorithms developed had good predictive ability and the errors identified fall within an acceptable range in comparison to other mapping studies [14]. Demographic information, other than age and sex, was not used during the mapping process. This was a deliberate choice appreciating that future use of the mapping algorithms will be maximized by the algorithms only requiring demographic information on age and sex [4].

The study had limitations. Given that the study relies on data collected within a pilot trial, a modest sample

Table 5 Mapping Models from the TOP to the EQ-5D-5 $L$ and the ICECAP-A

\begin{tabular}{lll}
\hline & EQ-5D-5 L & ICECAP-A \\
\hline Model & OLS (2) & OLS (2) \\
Intercept & $0.463^{\mathrm{b}}$ & $0.348^{\mathrm{b}}$ \\
Overall Quality of Life & -0.005 & $0.014^{\mathrm{a}}$ \\
Physical Health Status & 0.010 & -0.002 \\
Psychological Health Status & $0.024^{\mathrm{b}}$ & $0.015^{\mathrm{a}}$ \\
AIC & -52.030 & -70.213 \\
BIC & -42.402 & -60.586 \\
Adjusted R & 0.298 & 0.335 \\
RMSE (external sample) & 0.167 & 0.151 \\
MAE (external sample) & 0.123 & 0.123 \\
\hline
\end{tabular}

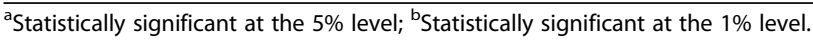
AIC Akaike information criterion, BIC Bayesian information criterion, MAE mean absolute error, OLS ordinary least squares, RMSE root mean squared error size was used. To overcome the sample size issue, observations were pooled from two time-periods but could have led to assumptions of independence being violated between the observations and lead to ungeneralizable results. However, appropriate techniques were applied to account for within-subject dependence.

Although the sample size was effectively doubled and the dependence accounted for, the pooled dataset did not produce better results relative to the algorithms created from the normal estimation dataset. Studies, however, have been conducted with smaller samples [39, 40]. The errors reported in this study are also not significantly different to ones reported within other studies [14]. At first glance, the sample may not appear representative of the general population but was generally representative of a UK OST population with a high number of men, unemployed and people with mainly white ethnicity. The EQ-5D-5 L and ICECAP-A had maximum scores of 1. Initial regression analysis identified results that led to scores greater than one. This meant that the upper boundary had to be censored to 1 . The main drawback with this approach is that the mean, RMSE and MAE scores are underestimated however there was no alternative solution as mean values above 1 are not possible.

Although, there are no published studies that map to the measures presented, there are many with other clinical measures that have mapped to the EQ-5D, which can offer a point of comparison. This study showed that applying an alternative regression specification such as the tobit regression did not improve the results, the OLS models were demonstrators of goodness of fit. This concords with other studies [41-44]. The predicted EQ-5D$5 \mathrm{~L}$ scores generated during the mapping process were across a much smaller spread than the observed EQ-5D- 


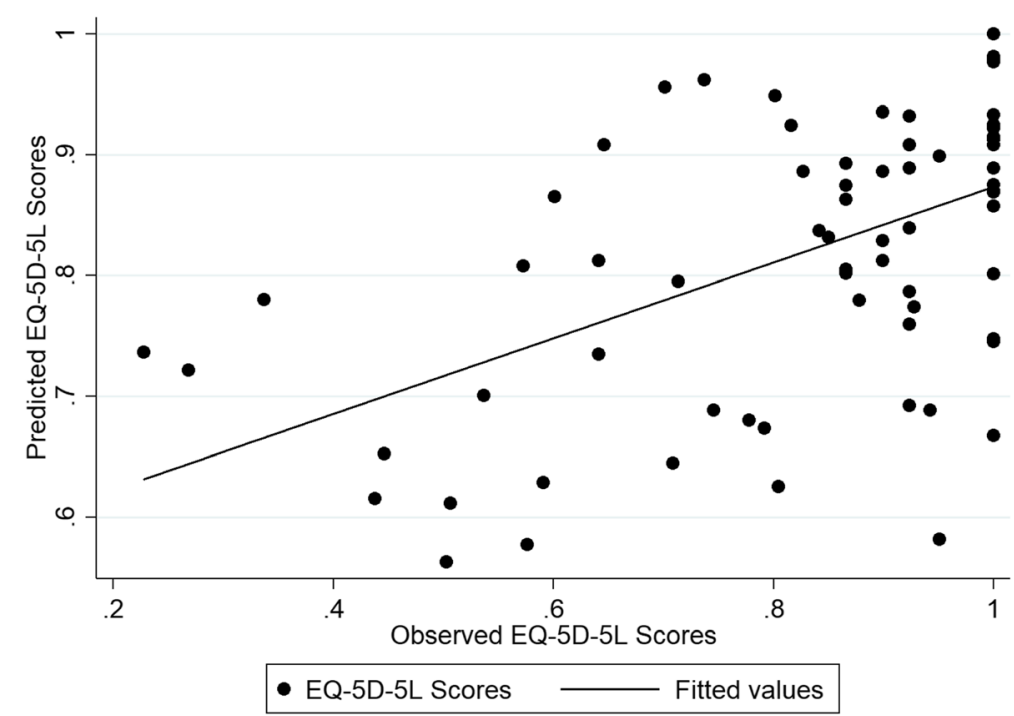

Fig. 5 The observed vs predicted scores of the EQ-5D-5 L mapped from the TOP based on Model 2

$5 \mathrm{~L}$ scores. This has also been seen to be the case in various other studies [41, 42].

As the results were generated from a fairly small sample size, it would be useful to validate the algorithms using a larger sample. It would also be important to conduct research into how the chosen algorithms would influence QALYs and cost-effectiveness decisions in the realm of mental health.

\section{Conclusion}

The application of the ICECAP-A could have the ability to capture mental health related quality of life outside the utility framework. In relation to mental health, recovery entails a variety of things, drug control, physical and mental health [45]. Mapping from a condition-specific measure to a traditional generic preference-based measure could miss out these key drivers within the recovery process. It is important to capture these impacts on an individual beyond the bounds of health and utilize tools such as the ICECAP-A wellbeing measure. The use of the TOP clinical measure is common practice particularly within the context of UK specialist drug treatment. Having these algorithms available provides the potential to estimate incremental QALYs and wellbeing outcomes using routinely collected data, and thus provides a framework for estimating the cost-effectiveness of alternative therapy options.

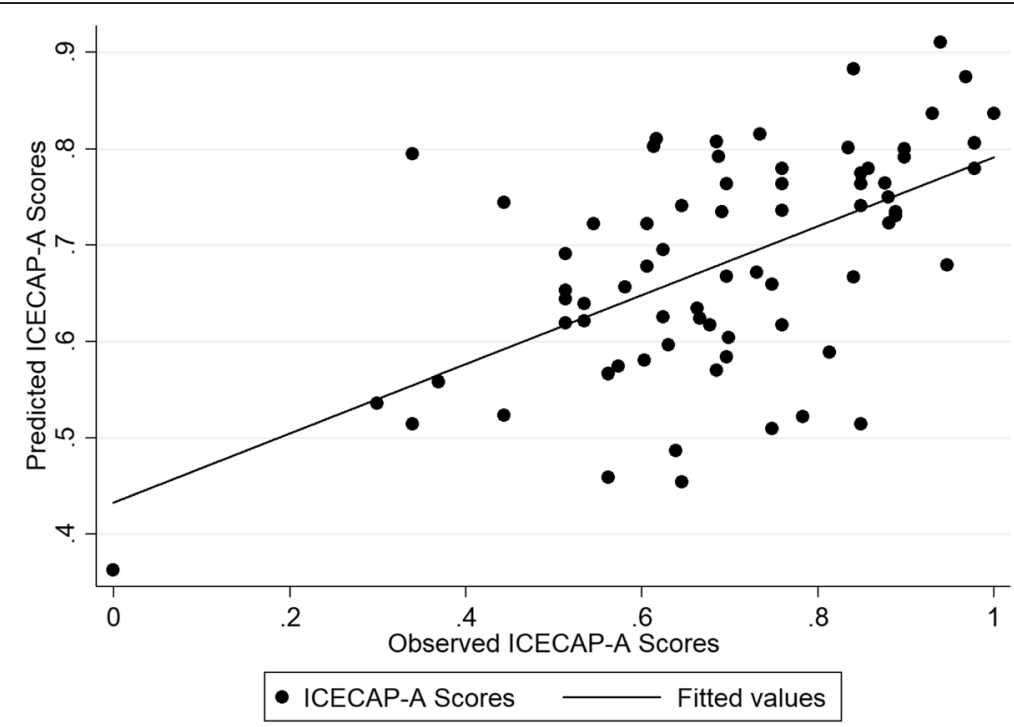

Fig. 6 The observed vs predicted scores of the ICECAP-A mapped from the TOP based on Model 2 


\section{Additional Files}

Additional file 1: Table S1. Descriptive statistics of the generic and condition-specific measures in the estimation and validation datasets. Statistics describing the features of the EQ- 5D-5 L, ICECAP-A, TOP, LDQ and the CORE-OM for the estimation and validation datasets. (DOCX $16 \mathrm{~kb}$ )

Additional file 2: Table S2. Model performance of the Internal Validation Sample Mapping from the CORE-OM to the EQ- 5D-5 L and the ICECAP-A. Results for each model when mapping from the CORE-OM to the EQ-5D and the ICECAP-A using the internal validation sample. (DOCX $17 \mathrm{~kb}$ )

Additional file 3: Table S3. Model performance of the External Validation Sample Mapping from the CORE-OM to the EQ- 5D-5 L and the ICECAP-A. Results for each model when mapping from the CORE-OM to the EQ-5D and the ICECAP-A using the external validation sample. (DOCX $17 \mathrm{~kb}$ )

Additional file 4: Table S4. Model performance of the best fitting models mapping from the CORE-OM to the ICECAP-A and the EQ-5D-5 L using the external validation sample. Results for the best fitting models, models 2 and 3, when mapping from the CORE-OM to the EQ-5D and the ICECAP-A using the external validation sample. (DOCX $14 \mathrm{~kb}$ )

Additional file 5: Table S5. Model performance of the Internal Validation Sample Mapping from the LDQ to the EQ- 5D-5 L and the ICECAP-A. Results for each model when mapping from the LDQ to the EQ-5D and the ICECAP-A using the internal validation sample. (DOCX $15 \mathrm{~kb}$ )

Additional file 6: Table S6 Model performance of the External Validation Sample Mapping from the LDQ to the EQ- 5D-5 L and the ICECAP-A. Results for each model when mapping from the LDQ to the EQ-5D and the ICECAP-A using the external validation sample. (DOCX $15 \mathrm{~kb}$ )

Additional file 7: Table S7 Model performance of the best fitting models mapping from the LDQ to the ICECAP-A and the EQ-5D-5 L using the external validation sample. Results for the best fitting models, models 3 and 4, when mapping from the LDQ to the EQ-5D and the ICECAP-A using the external validation sample. (DOCX $14 \mathrm{~kb}$ )

Additional file 8: Table S8. Model performance of the Internal Validation Sample Mapping from the TOP to the EQ- 5D-5 L and the ICECAP-A. Results for each model when mapping from the TOP to the EQ-5D and the ICECAP-A using the internal validation sample. (DOCX 14 kb)

Additional file 9: Table S9. Model performance of the External Validation Sample Mapping from the TOP to the EQ- 5D-5 L and the ICECAP-A. Results for each model when mapping from the TOP to the EQ-5D and the ICECAP-A using the external validation sample. (DOCX $16 \mathrm{~kb}$ )

Additional file 10: Table S10. Model performance of the best fitting models mapping from the TOP to the ICECAP-A and the EQ-5D-5 L using the external validation sample. Results for the best fitting models, models 1 and 2, when mapping from the LDQ to the EQ-5D and the ICECAP-A using the external validation sample. (DOCX $14 \mathrm{~kb}$ )

\section{Abbreviations}

AIC: Akaike Information Criterion; BIC: Bayesian Information Criterion; COREOM: Clinical Outcomes in Routine Evaluation - Outcome Measure; EQ-5D5 L: EuroQol - 5 Dimensions- 5 Levels; HRQOL: Health-Related Quality Of Life; ICECAP-A: ICEpop CAPability measure for Adults; LDQ: Leeds Dependence Questionnaire; MAE: Mean Absolute Error.; n: Number of patients; NICE: National Institute for Health and Care Excellence; OLS: Ordinary Least Squares; OST: Opiate Substitution Treatment; QALY: Quality-Adjusted Life Years; RCT: Randomised Control Trial; RMSE: Root-Mean-Squared-Error; SD: Standard Deviation; TOP: Treatment Outcomes Profile

\section{Acknowledgments}

We would like to thank everyone who participated in the study and to the community drug and alcohol teams located at Leicester (Leicestershire Partnership NHS Trust) and Solihull (Birmingham \& Solihull Mental Health NHS Foundation Trust).

\section{Funding}

The study was funded under the National Institute for Health Research (NIHR) under its Research for Patient Benefit (RfPB) Programme (Grant Reference Number PB-PG-0610-22392).

\section{Availability of data and materials}

The datasets used and/or analysed during the current study are available from the corresponding author on reasonable request.

\section{Authors' contributions}

JP led on the analysis of the data with support from IG and EF. ED is the chief investigator for the project. ED, AC, EF and NF contributed to the design of the study. All authors drafted the manuscript and read and approved the final manuscript.

\section{Ethics approval and consent to participate}

The study received ethics approval from the National Research Ethics Committee: The Black Country (REC number: 12/WM/0046; Approved 08/02/ 2012). Written, informed consent to participate in the study was obtained from all participants.

\section{Competing interests}

The authors declare that they have no competing interests.

\section{Publisher's Note}

Springer Nature remains neutral with regard to jurisdictional claims in published maps and institutional affiliations.

\section{Author details}

${ }^{1}$ Health Economics Unit, Institute of Applied Health Research, Public Health Building, University of Birmingham, B15 2T, Birmingham, UK. ${ }^{2}$ Research and Innovation Department, Birmingham \& Solihull Mental Health NHS Foundation Trust, Birmingham, UK. ${ }^{3}$ Addictions Department, Institute of Psychiatry, Psychology \& Neuroscience, King's College London, London, UK. ${ }^{4}$ School of Psychology, University of Birmingham, Birmingham, UK. ${ }^{5}$ Department of Primary Care and Population Health, University College London, London, UK. ${ }^{6}$ Melbourne School of Population and Global Health, University of Melbourne, Melbourne, Australia.

Received: 22 March 2018 Accepted: 7 May 2018

Published online: 30 May 2018

\section{References}

1. Morris S, Devlin N, Parkin D. Economic analysis in health care. Chichester: Wiley; 2007

2. Coast J. Maximisation in extra-welfarism: a critique of the current position in health economics. Soc Sci Med. 2009;69(5):786-92.

3. Drummond MF, Sculpher MJ, Claxton K, Stoddart GL, Torrance GW. Methods for the economic evaluation of health care programmes. Oxford: Oxford university press; 2005.

4. Chuang L, Whitehead SJ. Mapping for economic evaluation. Br Med Bull. 2012;101:1-15. doi: https://doi.org/10.1093/bmb/ldr049.

5. National Institute for Health and Care Excellence (NICE). Guide to the methods of technology appraisal. London: NICE; 2008.

6. Orford J. Power, powerlessness and addiction. Cambridge: Cambridge University Press; 2013.

7. Sen A. Capability and well-being. The quality of life. Oxford: Oxford University Press; 1993.

8. Al-Janabi H, Flynn TN, Coast J. Development of a self-report measure of capability wellbeing for adults: the ICECAP-A. Qual Life Res. 2012;21(1):167-76.

9. Goranitis I, Coast J, Day E, Copello A, Freemantle N, Seddon J, et al. Measuring health and broader well-being benefits in the context of opiate dependence: the psychometric performance of the ICECAP-A and the EQ5D-5L. Value Health. 2016;19(6):820-8

10. Day E, Copello A, Seddon JL, Christie M, Bamber D, Powell C, et al. Pilot study of a social network intervention for heroin users in opiate substitution treatment: study protocol for a randomized controlled trial. Trials. 2013;14(1):264.

11. Day E, Copello A, Seddon JL, Christie M, Bamber D, Powell C, Bennett C, Akhtar S, George S, Ball A, Frew E, Goranitis I, Freemantle N. A pilot feasibility randomised controlled trial of an adjunct brief social network intervention in opiate substitution treatment services. BMC Psychiatry. 2018;18(1):8. 
12. Baio G. Bayesian methods in health economics. Chapman and Hall/CRC Press. Boca Raton, FL, US. 2012.

13. Petrou S, Rivero-Arias O, Dakin H, Longworth L, Oppe M, Froud R, et al. Preferred reporting items for studies mapping onto preference-based outcome measures: the MAPS statement. Health Qual Life Outcomes. 2015b;13(1):106.

14. Brazier JE, Yang Y, Tsuchiya A, Rowen DL. A review of studies mapping (or cross walking) non-preference based measures of health to generic preference-based measures. Eur J Health Econ. 2010;11(2):215-25.

15. Petrou S, Rivero-Arias O, Dakin H, Longworth L, Oppe M, Froud R, et al. The MAPS reporting statement for studies mapping onto generic preferencebased outcome measures: explanation and elaboration PharmacoEconomics. 2015a;33(10):993-1011.

16. Evans C, Connell J, Barkham M, Margison F, McGrath G, Mellor-Clark J, et al. Towards a standardised brief outcome measure: psychometric properties and utility of the CORE-OM. Br J Psychiatry. 2002 Jan;180:51-60.

17. Barkham M, Mellor-Clark J, Connell J, Cahill J. A CORE approach to practicebased evidence: a brief history of the origins and applications of the COREOM and CORE system. Couns Psychother Res. 2006;6(1):3-15.

18. Raistrick D, Bradshaw J, Tober G, Weiner J, Allison J, Healey C. Development of the Leeds dependence questionnaire (LDQ): a questionnaire to measure alcohol and opiate dependence in the context of a treatment evaluation package. Addiction. 1994;89(5):563-72.

19. Raistrick R, Bradshaw J, Tober G, Weiner J, Allison J, Healey C. Development of the Leeds dependence questionnaire (LDQ): a questionnaire to measure alcohol and opiate dependence in the context of a treatment evaluation package. Addiction. 1994;89(5):563-572.

20. Marsden J, Farrell M, Bradbury C, Dale-Perera A, Eastwood B, Roxburgh M, et al. Development of the treatment outcomes profile. Addiction. 2008;103(9): $1450-60$.

21. National Treatment Agency for Substance Misuse. Treatment Outcomes Profile (TOP) [Internet]; 2017. Available from: http://www.nta.nhs.uk/ healthcare-TOP.aspx.

22. Herdman M, Gudex C, Lloyd A, Janssen M, Kind P, Parkin D, et al. Development and preliminary testing of the new five-level version of EQ-5D (EQ-5D-5L). Qual Life Res. 2011;20(10):1727-36.

23. Devlin N, Shah K, Feng Y, Mulhern B, van Hout B. Valuing health-related quality of life: an EQ-5D-5L value set for England.. 2016.

24. Flynn TN, Huynh E, Peters TJ, Al-Janabi H, Clemens S, Moody A, et al. Scoring the Icecap-a capability instrument. Estimation of a UK general population tariff. Health Econ. 2015;24(3):258-69.

25. Longworth L, Rowen D. Mapping to obtain EQ-5D utility values for use in NICE health technology assessments. Value Health. 2013;16(1):202-10.

26. Crott R, Briggs A. Mapping the QLQ-C30 quality of life cancer questionnaire to EQ-5D patient preferences. Eur J Health Econ. 2010;11(4):427-34.

27. McKenzie L, Van Der Pol M. Mapping the EORTC QLQ C-30 onto the EQ-5D instrument: the potential to estimate QALYs without generic preference data. Value Health. 2009;12(1):167-71

28. Pinedo-Villanueva RA, Turner D, Judge A, Raftery JP, Arden NK. Mapping the Oxford hip score onto the EQ-5D utility index. Qual Life Res. 2013;22(3):665-75.

29. Gray AM, Clarke PM, Wolstenholme JL, Wordsworth S. Applied methods of cost-effectiveness analysis in healthcare. Oxford: OUP; 2010.

30. Mitchell PM, Roberts TE, Barton PM, Pollard BS, Coast J. Predicting the ICECAP-O capability index from the WOMAC osteoarthritis index: is mapping onto capability from condition-specific health status questionnaires feasible? Med Decis Mak. 2013;33(4):547-57.

31. Dakin H, Gray A, Murray D. Mapping analyses to estimate EQ-5D utilities and responses based on Oxford knee score. Qual Life Res. 2013;22(3):683-94.

32. Skaltsa K, Longworth L, Ivanescu C, Phung D, Holmstrom S. Mapping the FACT-P to the preference-based EQ-5D questionnaire in metastatic castration-resistant prostate Cancer. Value Health. 2014;17(2):238-44.

33. Sullivan PW, Ghushchyan V. Mapping the EQ-5D index from the SF-12: US general population preferences in a nationally representative sample. Med Decis Mak. 2006;26(4):401-9.

34. Young TA, Mukuria C, Rowen D, Brazier JE, Longworth L. Mapping functions in health-related quality of life: mapping from two cancer-specific health-related quality-of-life instruments to EQ-5D-3L. Med Decis Mak. 2015;35(7):912-26.

35. Rivero-Arias O, Ouellet M, Gray A, Wolstenholme J, Rothwell PM, LuengoFernandez R. Mapping the modified Rankin scale (mRS) measurement into the generic EuroQol (EQ-5D) health outcome. Med Decis Mak. 2010;30(3):341-54.
36. Khan KA, Madan J, Petrou S, Lamb SE. Mapping between the Roland Morris questionnaire and generic preference-based measures. Value Health. 2014; 17(6):686-95.

37. Marra CA, Woolcott JC, Kopec JA, Shojania K, Offer R, Brazier JE, et al. A comparison of generic, indirect utility measures (the HUI2, HUI3, SF-6D, and the EQ-5D) and disease-specific instruments (the RAQoL and the HAQ) in rheumatoid arthritis. Soc Sci Med. 2005;60(7):1571-82.

38. Bray JW, Aden B, Eggman AA, Hellerstein L, Wittenberg E, Nosyk B, et al. Quality of life as an outcome of opioid use disorder treatment: a systematic review. J Subst Abus Treat. 2017;76:88-93.

39. Young MK, Ng S, Mellick G, Scuffham PA. Mapping of the PDQ-39 to EQ-5D scores in patients with Parkinson's disease. Qual Life Res. 2013;22(5):1065-72.

40. Kontodimopoulos N, Aletras VH, Paliouras D, Niakas D. Mapping the cancerspecific EORTC QLQ-C30 to the preference-based EQ-5D, SF-6D, and 15D instruments. Value Health. 2009:12(8):1151-7.

41. Cheung YB, Luo N, Ng R, Lee CF. Mapping the functional assessment of Cancer therapy-breast (FACT-B) to the 5-level EuroQoL Group's 5-dimension questionnaire (EQ-5D-5L) utility index in a multi-ethnic Asian population. Health Qual Life Outcomes. 2014;12(1):180.

42. Brazier J, Connell J, Papaioannou D, Mukuria C, Mulhern B, Peasgood T, et al. A systematic review, psychometric analysis and qualitative assessment of generic preference-based measures of health in mental health populations and the estimation of mapping functions from widely used specific measures. Health Technol Assess. 2014;18(34):vii,viii, xiii-xxv):1-188.

43. Hawton A, Green C, Telford C, Wright D, Zajicek J. The use of multiple sclerosis condition-specific measures to inform health policy decisionmaking: mapping from the MSWS-12 to the EQ-5D. Mult Scler J. 2012;18(6): 853-61.

44. Kim H, Kim D, Jang EJ, Lee M, Song HJ, Park S, et al. Mapping health assessment questionnaire disability index (HAQ-DI) score, pain visual analog scale (VAS), and disease activity score in 28 joints (DAS28) onto the EuroQol-5D (EQ-5D) utility score with the KORean observational study network for arthritis (KORONA) registry data. Rheumatol Int. 2016;36(4):505-13.

45. Goranitis I, Coast J, Day E, Copello A, Freemantle N, Frew E. Maximizing health or sufficient capability in economic evaluation? A methodological experiment of treatment for drug addiction. Med Decis Mak. 2017;37(5): $498-511$.

Ready to submit your research? Choose BMC and benefit from

- fast, convenient online submission

- thorough peer review by experienced researchers in your field

- rapid publication on acceptance

- support for research data, including large and complex data types

- gold Open Access which fosters wider collaboration and increased citations

- maximum visibility for your research: over $100 \mathrm{M}$ website views per year

At BMC, research is always in progress.

Learn more biomedcentral.com/submissions 